\title{
Deformidad tipo Checkrein del Hállux y dedos menores del pie sin fractura previa asociada.
}

\author{
DOI: http//dx.doi.org/10.37315/SOTOCAV20202825544 \\ RODRIGUEZ COLLELL JR. ${ }^{1}$, BLASCO SERRA A. ${ }^{2}$ RODRIGUEZ-PINO L ${ }^{3}$, MIFSUT MIEDES D. ${ }^{1,3}$. \\ 1 SERVICIO DE CIRUGÍA ORTOPÉDICA Y TRAUMATOLOGÍA. HOSPITAL CLÍNICO-MALVARROSA. VALENCIA, ESPAÑA. \\ 2 DEPARTAMENTO DE ANATOMÍA Y EMBRIOLOGÍA. UNIVERSITAT DE VALÈNCIA. \\ 3 DEPARTAMENTO DE CIRUGÍA. UNIVERSITAT DE VALÈNCIA.
}

\section{Resumen.}

Presentamos una revisión bibliográfica y la descripción de la técnica quirúrgica en la deformidad tipo Checkrein del hállux y dedos menores del pie. Esta deformidad dinámica provoca a los pacientes una dificultad significativa para caminar y les impide practicar cualquier deporte ya que en la fase de apoyo de la marcha los dedos del pie se ven forzados a una flexión plantar máxima y terminan atrapados debajo del pie. En los casos en que no se asocia a fractura ósea la sospecha clínica causante de la lesión es un síndrome compartimental subclínico. El tratamiento descrito en este trabajo consiste en una plastia en Z y la aplicación de una sutura pulvertaft en el flexor largo del hállux. En el resto de dedos no requieren ningún gesto quirúrgico añadido, al corregirse la deformidad a nivel retromaleolar.

Palabras clave: Checkrein deformity, Pulvertaft suture. Dynamic deformity of the great toe.

\section{Summary.}

We present a bibliographic review and a description of the surgical technique in checkrein deformity of the hallux and lesser toes. This dynamic deformity causes a significant difficulty in walking and prevents patients from practicing any sport, since in the stance phase of gait toes are forced into maximum plantar flexion and end up trapped under the foot. In cases in which this is not associated with a bone fracture, the clinical suspicion causing the injury is a subclinical compartment syndrome. Treatment described in this work consists of a Z-plasty and the application of a pulvertaft suture in the flexor hallux longus. In the rest of the toes, any additional surgical procedure is not needed, as the deformity is corrected at the retromalleolar level.

\section{Correspondencia:}

Juan Ramón Rodríguez Collell

rocojura@hotmail.com 
RODRIGUEZ-COLLELL JR, Y COLS. Deformidad tipo Checkrein del Hallux y dedos menores del pie sin fractura asociada.

\section{Introducción}

La deformidad dinámica posicional del Hállux o Checkrein Deformity, se debe a un atrapamiento del Flexor Hallucis Longus ( $\mathrm{FHL}$ ), aunque ocasionalmente puede afectarse el Flexor Digitorum Longus (FDL), provocando una contractura en flexión del primer dedo del pie, asociada o no, a flexión del resto de dedos pequeños ${ }^{1-3}$. La deformidad aumenta con la dorsiflexión del tobillo y disminuye o desaparece con la flexión plantar.

Esta patología fue descrita por primera vez por Clawson en $1974^{1}$, al observarla tras una fractura de tibia, desde entonces se han descrito casos tras fracturas de calcáneo ${ }^{2}$, de tibia y peroné ${ }^{3-9}$, utilización del peroné como injerto ${ }^{10}$, fracturas epifisiolisis de tibia distal ${ }^{11}$, y en pocos casos tras un traumatismo sobre partes blandas sin fractura ${ }^{12}$.

Sin un mecanismo patogénico claro, diferentes teorías ${ }^{3-5}$ se han propuesto en la literatura para tratar de justificar el origen de esta condición, aunque por el momento ninguna ha demostrado ser la verdadera.

El interés de nuestro estudio es precisamente que en nuestro caso no hubo fractura previa como desencadenante de la lesión.

Presentamos un caso clínico de un paciente que sufrió una deformidad Checkrein del hállux y del $2^{\circ}$ dedo pequeño del pie derecho, tras sufrir un traumatismo directo sobre partes blandas en su pierna derecha, sin fractura asociada, fue tratado mediante $\mathrm{z}$ plastia y sutura pulvertaft del flexor hallucis longus, con resultado satisfactorio.

\section{Caso Clínico}

Paciente varón de 37 años de edad, que sufrió un accidente de tráfico "coche contra moto", quedando su pierna derecha atrapada entre el parachoques del coche y el lateral de la moto. Fue diagnosticado de una herida contusa en cara antero-interna de la pierna derecha, realizándose una limpieza y Friedrich de la herida. Al cabo de un año consultó por una deformidad progresiva en flexión plantar del hállux y dedos menores del pie derecho cuando el tobillo se encontraba en posición de dorsiflexión (Fig. 1).

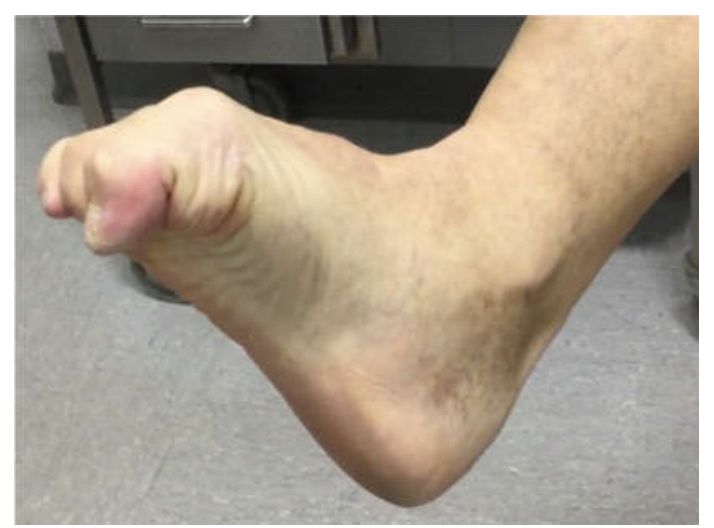

Figura 1: Imagen clínica donde se observa la deformidad del Hallux y resto de dedos.
Esta deformidad dinámica le dificultaba caminar y le impedía practicar ningún deporte, ya que en la fase de apoyo del pie, los dedos se veían forzados a permanecer en una flexión plantar máxima, quedando atrapados bajo el pie. Sin embargo al colocar el tobillo en flexión plantar la flexo-extensión activa de los dedos del pie era completa.

En el estudio por rx simple y RM (Resonancia Magnética) no se observó ninguna lesión músculo-tendinosa ni ósea (Fig. 2).

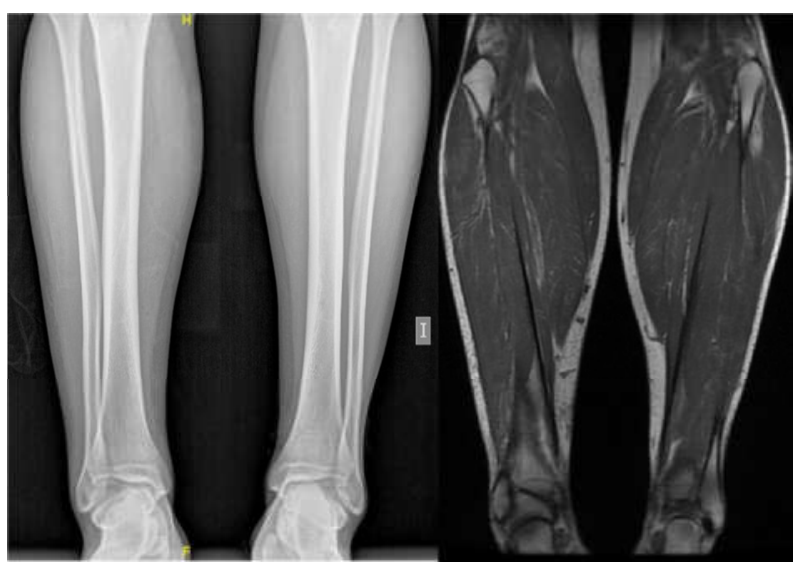

Figura 2: Imagen de rx simple y RNM donde se descartan fracturas y lesiones en partes blandas

Fue diagnosticado de Deformidad tipo Checkrein del Hállux y de los dedos menores.

El paciente fue intervenido quirúrgicamente, realizándose un abordaje retromaleolar medial, se identificó el tendón del flexor largo del hállux (FHL) (Fig. 3) y se realizó una tenotomía en $Z$ del tendón.

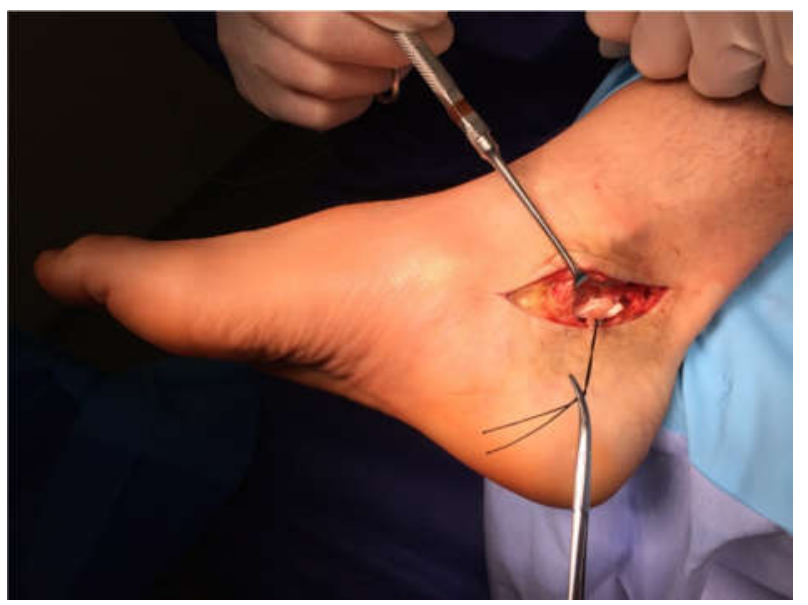

Figura 3: Identificación del tendón flexor largo del hállux en abordaje retromaleolar.

Se dio un punto de referencia a los extremos tendinosos resultantes de manera que el hállux quedara en flexoextensión neutra, mientras el tobillo se mantenía también en flexo-extensión neutra. A continuación se realizó una sutura de la tenotomía en "pulvertaft" (Fig. 4). 

asociada.

No se realizó ningún gesto sobre los tendones de los dedos menores.

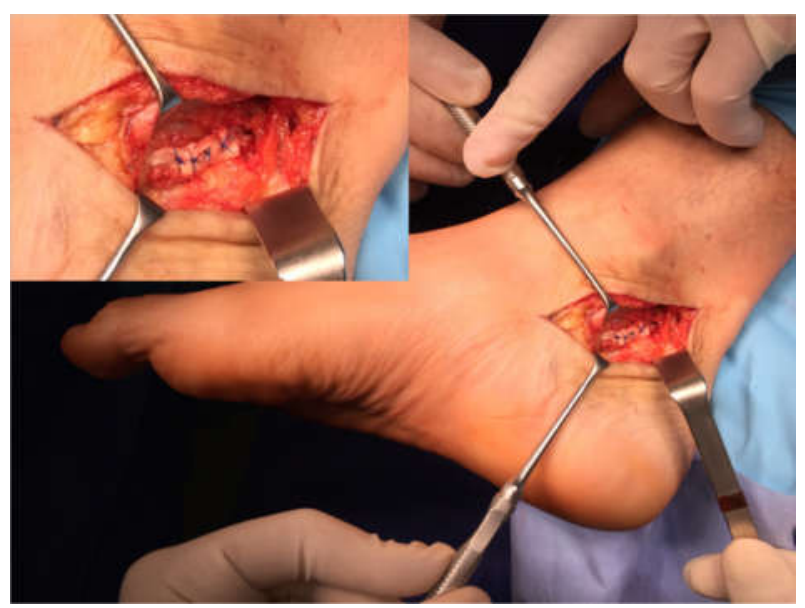

Figura 4: sutura de la tenotomía en "pulvertaft"

Tras la intervención, el paciente permaneció inmovilizado con una férula durante 3 semanas con el tobillo en posición neutra. Tras un breve periodo de rehabilitación (2 semanas), la recuperación del paciente fue completa, recuperando la flexo-extensión activa del primer dedo y de los dedos pequeños del pie cuando el tobillo permanecía en dorsiflexión neutra (Fig. 5).

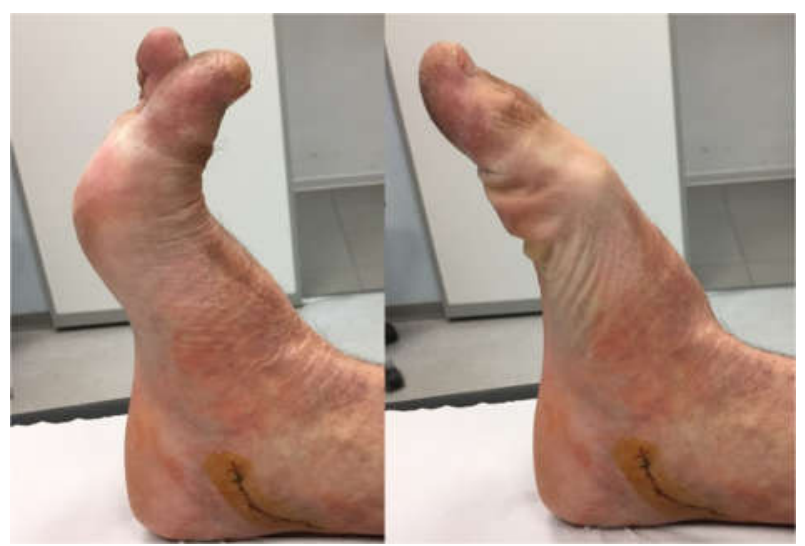

Figura 5: Resultado final clínico con movilización completa de los dedos.

\section{Discusión}

La deformidad tipo Checkrein es una alteración en flexión dinámica del tendón de $\mathrm{FHL}$ y generalmente ocurre después de una fractura de tibia. Cuando el tobillo se dorsiflexiona pasivamente, la deformidad de la articulación interfalángica se vuelve más prominente. Se ha asociado en ocasiones a deformidades de los dedos menores, debido a que el tendón $\mathrm{FHL}$ tiene alguna interconexión con los tendones del FDL.

Desde que Clawson ${ }^{1}$ describió por primera vez los dedos en garra después de fracturas de tibia en 1974, la deformidad por Checkrein ha sido observada en otras circunstancias.
Carr et $\mathrm{al}^{3}$ observó atrapamiento del tendón de $\mathrm{FHL}$ después de fracturas del calcáneo, y la tomografía computarizada demostró el atrapamiento del tendón en fragmentos de fractura. Leitschuh et $\mathrm{al}^{4}$ observó una deformidad de flexión del hállux secundaria a atrapamiento del tendón de $\mathrm{FHL}$ después de una fractura del peroné. Se encontró que el tendón estaba adherido en el foco de la fractura consolidada.

La masa muscular del FHL es mayor que la del FDL detrás de la tibia distal, pudiendo ser una de las razones por las que el FHL está involucrado más comúnmente.

Una deformidad de Checkrein típica se ha descrito después de una fractura concomitante de peroné distal ${ }^{5,7,9}$.

Lee et al ${ }^{12}$ describieron 11 casos de Checkrein Deformity pero solo uno de ellos no estaba asociado a una fractura previa.

Las dos teorías causales más extendidas en la actualidad son:

1. Un atrapamiento del FHL o del EHL en el tejido cicatricial de partes blandas o en el callo de fractura secundario a una lesión por traumatismo de alta energía.

2. Un aumento de presión compartimental (que algunos autores describen como subclínico, ya que la mayoría de pacientes no llegan a sufrir un síndrome compartimental agudo establecido) 5 que condicionaría una lesión en la unión musculo-tendinosa del $\mathrm{FHL}$ o del $\mathrm{EHL}$ con el consiguiente atrapamiento y posterior instauración de la deformidad.

La primera teoría podría justificarse cuando se produce una fractura, pero el atrapamiento tendinoso daría lugar a una deformidad fija, no dinámica, debido a las adherencias del tendón; pero como hemos visto en nuestro caso, cuando no existe fractura la teoría que explicaría con mayor exactitud la deformidad sería la segunda, ya que la deformidad es dinámica por retracción del vientre muscular.

En cuanto a la técnica quirúrgica, el tendón flexor hallucis longus surge de los dos tercios inferiores de la superficie posterior del peroné y la membrana interósea. Lee et al. ${ }^{12}$ describieron dos tipos de reparación quirúrgica para la deformidad de Checkrein, uno con liberación de adherencias y alargamiento de la plastia $Z$ en la unión musculotendinosa por encima del tobillo en el sitio de la fractura y la otra técnica que implica el alargamiento de la flexor hallucis longus en el mediopie. Algunos autores refieren una mejor evolución con estos últimos procedimientos ${ }^{7}$, mientras que los procedimientos más proximales tenían diferentes grados de recurrencia en la formación de nuevas adherencias.

Sanhudo y Lompa $^{10}$, y $\operatorname{Sinnett}^{13}$ sugirieron que el alargamiento del tendón a nivel del mediopié es mejor que retromaleolar al tobillo, además, el alargamiento por separado del FHL y FDL a veces es necesario, y la operación en el pie permite la división de las interconexiones entre FHL y FDL, por el contrario, la 

asociada.

intervención en el sitio de la fractura requiere una gran incisión con disección de estructuras neurovasculares.

Según Feeney $\mathrm{M}^{14}$ y Lee $\mathrm{H}^{12}$, el alargamiento del $\mathrm{FHL}$ es suficiente para tratar la deformidad tipo Checkrein asociada a deformidad de los dedos menores, ya que existen interconexiones tendinosas entre el FHL y el FDL, siendo preferible realizar el alargamiento del tendón de $\mathrm{FHL}$ por plastia en $Z$ por encima del tobillo. En nuestro caso, realizamos el alargamiento a nivel proximal, y no se realizó ningún gesto sobre el FDL, consiguiendo aun así, corrigir la deformidad del resto de los dedos. El motivo de la liberación aislada del $\mathrm{FHL}$ se debió a que las interconexiones del FHL con el FDL se producen a nivel distal al retropié.
En todo caso, la movilización precoz de los dedos de los pies después de la lesión es importante, especialmente cuando se asocia a fractura.

\section{Conclusiones}

La deformidad tipo Checkrein es una patología poco frecuente pero no solo secundaria a fracturas sino también a traumatismos de partes blandas, ya que suele asociarse un Síndrome compartimental subclínico.

La tenotomía de alargamiento aislada del FHL a nivel retromaleolar es suficiente para corregir la deformidad asociada de los dedos menores.

\section{Bibliografía}

1. Clawson D. Claw toes following tibial fracture. Clin Orthop Relat Res 1974; 103:47-8.

2. Kim SH, Lee KT, Smith RW, Park YU. Checkrein deformity secondary to entrapment of FHL after talus fracture: a case report. Foot Ankle Int 2010;31:336-8.

3. Carr J. Complications of calcaneus fractures entrapment of the Flexor Hallucis Longus. J Orthop Trauma 1990; 4:166-8.

4. Leitschuh P, Zimmerman J, Uhorchak J, Arciero R, Bowser L. Hallux Flexion Deformity Secondary to Entrapment of the Flexor Hallucis Longus Tendon after Fibular Fracture. Foot Ankle Int 1995; 16:232-5.

5. Carranza-Bencano A, Gómez-Arroyo J, Fernández-Torres J. Hallux flexus deformity due to entrapment of the flexor hallucis longus tendon after an open fracture of the tibia and fibula. Foot Ankle Surg 2000; 6:133-5.

6. Holcomb T, Temple E, Barp E, Smith H. Surgical Correction of Checkrein Deformity after Malunited Distal Tibia Fracture: A Case Report. J Foot Ankle Surg 2014; 53:631-4.

7. Martínez Pérez C, Peralta Nieto J, Gil Monzó ER, Pérez Blasco AV, Borras Cebrián JC, Rodrigo Pérez JL. Prevalencia en nuestro medio de las deformidades dinámicas posicionales del hallux como secuela de las fracturas de tercio medio y distal de tibia. Rev Esp Osteoart 2016; 267( 51):129-133.

8. Gadhavi MV, Majmundar DD', Solanki RA. Checkrein Deformity of the Great toe Managed by Midfoot Flexor Hallucis Longus Z-Plasty: A Case Report . J Orthop Case Reports 2019; 9(2):18-20.

9. Yuen CLui T. Adhesion of flexor hallucis longus at the site of a tibial-shaft fracture- A cause of a checkrein deformity. Foot Ankle Surg 2015; 21:e23-6.

10. Sanhudo JA, Lompa PA. Checkrein deformity-flexor halluces tethering: two case reports. Foot Ankle Int 2002; 23: 799-800. 11.. 11. Rosenberg, GA; Sferra, JJ. Checkrein Deformity - Na Unusual Complication Associated with a Closed Salter-Harris Type II Ankle Fracture: A Case Report. Foot Ankle Int.;20(9):591-594, 1999.

12. Lee H, Kim J, Park S, Lee D, Park J, Wapner K. Treatment of checkrein deformity of the hallux. J Bone Joint Surg Br 2008; 90:1055-8.

13. Sinnett T, Rudge B, Clark C. A case of check-rein deformities of the great and lesser toes treated through exploration at the midfoot. Foot Ankle Surg 2015;21(1):e6-8.

14. Feeney M, Williams R, Stephens M. Selective lengthening of the proximal flexor tendon in the management of acquired claw toes. J Bone Joint Surg Br 2001; 83:335-8. 